\title{
Culex flavivirus infection in a Culex pipiens mosquito colony and its effects on vector competence for Rift Valley fever phlebovirus
}

\author{
Sandra Talavera ${ }^{1}$, Lotty Birnberg ${ }^{1}$, Ana I. Nuñez ${ }^{1}$, Francesc Muñoz-Muñoz ${ }^{2}$, Ana Vázquez ${ }^{3,4}$ and Núria Busquets ${ }^{1 *}$
}

\begin{abstract}
Background: Rift Valley fever is a mosquito-borne zoonotic disease that affects domestic ruminants and humans. Culex flavivirus is an insect-specific flavivirus that naturally exists in field mosquito populations. The influence of Culex flavivirus on Rift Valley fever phlebovirus (RVFV) vector competence of Culex pipiens has not been investigated.

Methods: Culex flavivirus infection in a Cx. pipiens colony was studied by Culex flavivirus oral feeding and intrathoracical inoculation. Similarly, vector competence of $C x$. pipiens infected with Culex flavivirus was evaluated for RVFV. Infection, dissemination, transmission rates and transmission efficiency of Culex flavivirus-infected and non-infected $C x$. pipiens artificially fed with RVFV infected blood were assessed.

Results: Culex flavivirus was able to infect $C$. pipiens after intrathoracically inoculation in $C X$. pipiens mosquitos but not after Culex flavivirus oral feeding. Culex flavivirus did not affect RVFV infection, dissemination and transmission in $C$. pipiens mosquitoes. RVFV could be detected from saliva of both the Culex flavivirus-positive and negative $C X$. pipiens females without significant differences. Moreover, RVFV did not interfere with the Culex flavivirus infection in CX. pipiens mosquitoes.

Conclusions: Culex flavivirus infected and non-infected CX. pipiens transmit RVFV. Culex flavivirus existing in field-collected CX. pipiens populations does not affect their vector competence for RVFV. Culex flavivirus may not be an efficient tool for RVFV control in mosquitoes.
\end{abstract}

Keywords: Rift Valley fever phlebovirus, Culex pipiens, Culex flavivirus, Transmission, Vector competence

\section{Background}

Culex flavivirus (CxFV) belongs to the genus Flavivirus (family Flaviviridae). The majority of viruses within this genus are transmitted horizontally between vertebrate hosts and hematophagous arthropods. However, some flaviviruses are considered to be vertebrate-specific while other group of viruses of this genus are insect-specific (ISFV) [1-3]. Circulation of ISFVs in natural mosquito populations is likely maintained by vertical transmission $[4,5]$. In Europe, several species of ISFV have been

\footnotetext{
* Correspondence: nuria.busquets@irta.cat

${ }^{1}$ IRTA, Centre de Recerca en Sanitat Animal (CReSA, IRTA-UAB), Campus de la Universitat Autònoma de Barcelona, 08193 Bellaterra, Spain

Full list of author information is available at the end of the article
}

detected in field mosquitoes from Italy, Portugal, Spain, the United Kingdom, the Czech Republic and Greece [6-10]. Sequences related to those viruses have been detected worldwide [11-14]. ISF RNA has also been detected in sand flies (family Psychodidae) in Algeria [2], Spain [3] and Portugal (GenBank: HM563684). Previous field studies in Spain suggested the existence of a large number of ISF $[3,15,16]$, though not completely characterized phylogenetically [10]. The circulation of ISFV in nature raises concerns regarding possible interactions with arthropod-borne flaviviruses [17] and even other arboviruses in vector populations. Co-infection studies with mosquito-borne flaviviruses (MBFV) and ISFV have been performed in order to gain a better understanding

(c) The Author(s). 2018 Open Access This article is distributed under the terms of the Creative Commons Attribution 4.0 International License (http://creativecommons.org/licenses/by/4.0/), which permits unrestricted use, distribution, and reproduction in any medium, provided you give appropriate credit to the original author(s) and the source, provide a link to the Creative Commons license, and indicate if changes were made. The Creative Commons Public Domain Dedication waiver (http://creativecommons.org/publicdomain/zero/1.0/) applies to the data made available in this article, unless otherwise stated. 
of any factor that could alter vector competence of mosquitoes for MBFV in both enzootic and epizootic transmission cycles [18]. Three studies were carried out to directly address potential co-infection exclusion effect between $\mathrm{CxFV}$ and other flaviviruses such as West Nile virus (WNV) [18-20]. However, no co-infection studies with other pathogenic viruses belonging to other genera have been performed, such as Rift Valley fever phlebovirus (RVFV).

Rift Valley fever (RVF) is a mosquito-borne zoonotic disease caused by RVFV (genus Phlebovirus, family Phenuiviridae). RVFV is transmitted by mosquito bites to a large number of hosts, both domestic and wild ruminants [21]. Described for the first time in 1931 in Kenya [22], RVFV has continuously caused outbreaks in animals and humans in several African countries [23]. In 2000, RVFV was first reported outside of Africa, i.e. in Saudi Arabia and Yemen [24], linking to the likelihood of a potential introduction of RVFV in Europe. The risk of RVFV introduction in Europe has been recently evaluated [25-28]. Results of a multiple criteria decision-making model study of key factors for RVF in Spain identified areas with high suitability for RVF outbreak occurrence in each month of the year [28]. Moreover, a previous study has shown that a Culex pipiens mosquito colony from Spain is able to transmit this virus [29]. Species of the genera Aedes and Culex are considered main vectors of RVFV [30]. Culex pipiens complex is considered as an efficient RVFV vector [31] including $C x$. pipiens and $C x$. quinquefasciatus which are ubiquitous mosquitoes in temperate and tropical regions, respectively [32].

It is relevant to understand ISF dynamics and their role in their mosquito hosts as potential control tool for vector-borne pathogens. To this end, the objectives of the present study were to evaluate (i) the CxFV infection in a $C x$. pipiens colony by oral feeding and intrathoracic inoculation and (ii) the role in vector competence of CxFV for RVFV infection, dissemination and transmission by $C x$. pipiens. All experiments were performed simulating environmental conditions of the season with high vector density and high suitability for RVF outbreak occurrence in the distribution area of the tested mosquito population.

\section{Methods}

\section{Mosquito populations}

One mosquito population of $C x$. pipiens pipiens and molestus hybrid form from Gavà (2012), Catalonia (northeastern Spain) was used. Molecular characterization of the $C x$. pipiens forms was performed for each individual involved in the RVFV vector competence assay as previously described [33]. The Cx. pipiens colony was reared in laboratory under environmental conditions: temperature, $26{ }^{\circ} \mathrm{C}: 22{ }^{\circ} \mathrm{C}$ (day:night); relative humidity
(RH) of $80 \%$; and a $14: 10$ h (L:D) photoperiod including two crepuscular cycles of $30 \mathrm{~min}$ to simulate dawn and dusk.

Before vector competence assays, the mosquito colony was tested for the presence of viruses, as described previously [29], to exclude other viral infections (species of Flavivirus, Alphavirus and Phlebovirus). In the last decade, other novel insect-specific viruses have been detected in field mosquitoes belonging to several families such us Bunyaviridae, Mesoniviridae, Reoviridae, Rhabdoviridae, Togaviridae and the newly recognized taxon of Negeviruses [34]. Prior to vector competence assays, the colony was also tested for the presence of these viruses using generic RT-nested-PCR (unpublished) and Wolbachia spp. by PCR [35]. The mosquito colony was found to be Wolbachia spp.-positive and negative for Flavivirus, Alphavirus, Phlebovirus, Bunyaviridae, Mesoniviridae, Reoviridae, Rhabdoviridae, Togaviridae and Negeviruses (data not shown).

\section{Virus strains}

The CxFV strain was detected in field-collected Culex pipiens mosquitoes captured in Huelva, Spain, in 2006, and isolated in $\mathrm{C6} / 36$ cells. To propagate the virus, C6/36 cells were incubated for $6-7$ days $\left(28{ }^{\circ} \mathrm{C}, 5 \% \mathrm{CO}_{2}\right)$ and viral particles were observed by electronic microscopy. As cytopathic effect was not observed, CxFV replication was detected in the supernatant using a modified real time RT-PCR [19] (see below). A monolayer of C6/36 cells was used to titrate CxFV. Briefly, eight wells were infected for each ten-fold dilution. Twenty microliters of inoculum and $150 \mu \mathrm{l}$ of minimum essential medium (Life Technologies, Carlsbad, CA, USA) supplemented with 2\% FBS (EuroClone SpA, Pero, Italy), $2 \mathrm{mM}$ L-glutamine, nonessential amino acids, $1000 \mathrm{U} / \mathrm{ml}$ of penicillin, $10 \mathrm{mg} / \mathrm{ml}$ of streptomycin and $500 \mathrm{U} / \mathrm{ml}$ of nystatin (all from SigmaAldrich, St. Louis, MO, USA), were added into each well as post-infection medium. Plates were incubated at $28{ }^{\circ} \mathrm{C}$ and $5 \%$ of $\mathrm{CO}_{2}$ for 7 days. Calculation of the viral titer was performed by virus detection in each well using real time RT-PCR. Ct-values ranged between 21.09-23.48 in the wells where the virus replicated. The 50\% tissue culture infective dose per milliliter (TCID50/ml) was calculated using the Reed \& Muench method [36].

The virulent RVF 56/74 strain (passages history [37] and one passage in C6/36 cells) was propagated in BHK-21 cells. The virus was titrated in Vero cells and cytopathic effect was observed. The 50\% tissue culture infective dose per milliliter (TCID50/ml) was also calculated using the Reed \& Muench method [36].

\section{CxFV infection in mosquitoes orally exposed}

Fourteen-day-old $C x$. pipiens females were exposed for $60 \mathrm{~min}$ to $\mathrm{CxFV}$ infected blood $(1: 2)$ at $4 \log _{10} \mathrm{TCID}_{50} /$ 
$\mathrm{ml}$ using the Hemotek feeder system. At $0,3,5,7$ and 10 days post-exposure (dpe), six fed females were harvested and frozen until analysis.

\section{CxFV intrathoracic inoculation in mosquitoes}

A group of $36 \mathrm{Cx}$. pipiens females, 2-3 days of age, were intrathoracically inoculated with $\mathrm{CxFV}$ at $4 \log _{10} \mathrm{TCID}_{50} /$ ml diluted 1:2 in Dulbecco's modified Eagle medium (DMEM). To study virus replication kinetics, these females were examined at $0,3,5,7,9$ and 11 days postinoculation (dpi). Bodies were analyzed from the 36 mosquitoes and saliva was harvested from all mosquitoes except from those corresponding to 0 dpi. Saliva was collected using a capillary technique as previously described [29]. As an inoculation control, a group of mosquitoes was inoculated with only DMEM.

\section{RVFV vector competence assay}

The ability of RVFV to infect, disseminate and be transmitted by $C x$. pipiens infected and non-infected with CxFV was evaluated by: infection rate (IR), disseminated infection rate (DIR), transmission rate (TR) and transmission efficiency (TE). IR refers to the proportion of mosquitoes with infected body among tested mosquitoes. DIR corresponds to the proportion of mosquitoes with infected legs/wings among the previously detected infected mosquitoes (i.e. body positive). TR represents the proportion of mosquitoes with infected saliva among mosquitoes with disseminated infection. TE represents the proportion of mosquitoes with infected saliva among the total number of mosquitoes tested [38].

Seven- to nine-day-old female mosquitoes that had never been blood-fed were used. Mosquitoes were reared and fed as previously described [29]. Culex pipiens intrathoracically inoculated with $\mathrm{CxFV}$ or with DMEM were tested for vector competence (VC) using a RVFV viral dose of $7.23 \log _{10} \mathrm{TCID}_{50} / \mathrm{ml}$. After the blood-feeding, $\mathrm{CO}_{2}$ was used to anesthetize the mosquitoes and fullyengorged females (FEF) were selected. The blood doped with RVFV was titrated in Vero cells. Ten percent of the specimens from each group were sacrificed and analyzed as a control of the inoculum. The rest of the mosquitoes were individually placed to cardboard cages (Watkins \& Doncaster, Leominster, UK).

FEF were fed with sucrose (10\%) ad libitum using soaked cotton pledgets. The presence of viral RNA in saliva was evaluated using two different approaches: FTA $^{\text {TM }}$ cards (GE Healthcare, Little Chalfont, UK) soaked with Manuka honey (Manuka Health New Zealand, Te Awamutu, New Zealand) and a blue alimentary colorant, at 4 and 14 dpe and the direct extraction of mosquitoes' saliva by capillarity at 14 dpe. At 4 and 14 dpe the FTA cards were left $24 \mathrm{~h}$ on the top of the mesh screen of all cardboard cages to allow the mosquito to feed on it. After FTA cards collection, they were resuspended in $0.3 \mathrm{ml}$ of PBS and stored at $-80{ }^{\circ} \mathrm{C}$ until tested. At 14 dpe, every mosquito was anesthetized with $\mathrm{CO}_{2}$ and dissected, and samples (legs/wings and bodies) were collected as previously described [29]. One hundred-fifty microliters from the saliva sample contained in DMEM medium were used for viral RNA extraction and the remaining $50 \mu \mathrm{l}$ were used for RVFV isolation in a Vero cells monolayer. Cells were incubated for 7 days $\left(37^{\circ} \mathrm{C}, 5 \% \mathrm{CO}_{2}\right)$ and the cytophatic effect was evaluated.

\section{Virus detection}

CxFV detection was performed using the real time RT-PCR protocol described by Bolling et al. [19] with minor modifications. The primer CxFV-F was modified as follows: 5'CTA CGC TCT TAA CAC AGT GA-3' and RT-qPCR was carried out using Quantitec SyBr Green RT-PCR kit (Qiagen, Hilden, Germany). Samples were amplified using a 7500 Fast Real-Time PCR System (Applied Biosystems, Foster City, CA, USA) programmed as follows: $50{ }^{\circ} \mathrm{C}$ for 10 min, $95{ }^{\circ} \mathrm{C}$ for $10 \mathrm{~min}, 45$ cycles at $95{ }^{\circ} \mathrm{C}$ for $15 \mathrm{~s}$ and at 57 ${ }^{\circ} \mathrm{C}$ for $35 \mathrm{~s}$. RVFV RNA was extracted and detected as previously described [29].

\section{Statistical analysis}

The frequency with which $\mathrm{CxFV}(+)$ and $\mathrm{CxFV}(-)$ mosquitoes get infected, disseminate, and transmit RVFV was compared by Fisher's exact test. Ct-values in mosquito bodies, legs/wings and saliva 14 dpe were compared between $\mathrm{CxFV}(+)$ and $\mathrm{CxFV}(-)$ groups by a non-parametric Mann-Whitney test as data were not normally distributed. Differences in Ct-values in $\mathrm{CxFV}$ inoculated mosquitoes among dpi were assessed by means of a multiple comparisons Kruskal-Wallis test. $P$-values $<0.05$ were considered statistically significant.

\section{Results \\ CxFV replication kinetics in orally exposed $C x$. pipiens}

No CxFV replication was detected in $C x$. pipiens exposed orally, suggesting that $C x$. pipiens mosquitoes are not susceptible to CxFV infection by oral exposure. Although no positive CxFV was recorded in any tested female mosquito on 3, 5, 7 and 10 dpe, CxFV could be detected in all mosquito samples collected on 0 dpe, demonstrating that all mosquitoes were exposed to the virus (Fig. 1).

\section{CxFV replication kinetics in Cx. pipiens intrathoracically inoculated}

Culex pipiens intrathoracically inoculated with CxFV showed viral replication. Results demonstrated a high percentage of $\mathrm{CxFV}$ infection detected at all time-points 


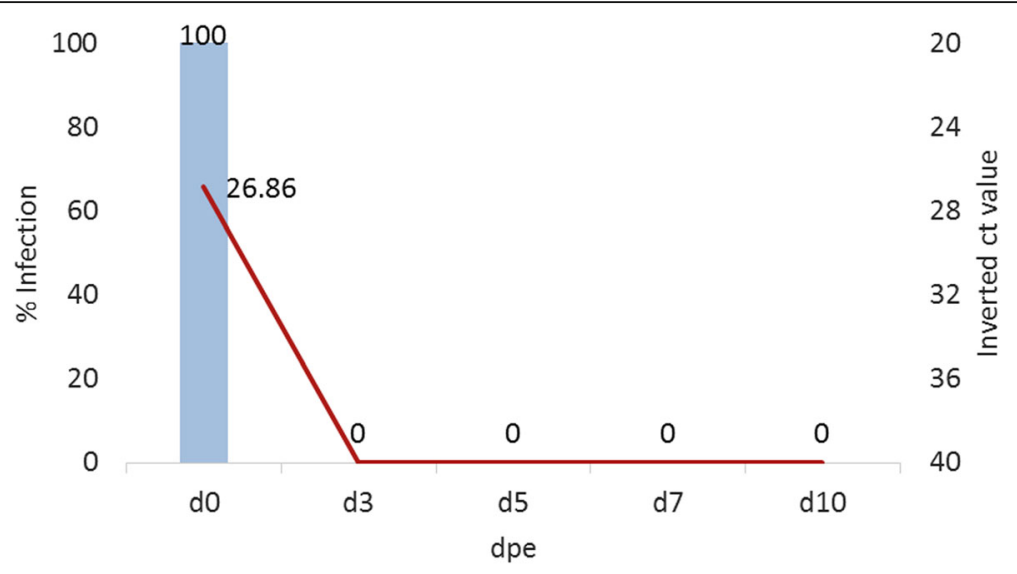

Fig. 1 CXFV replication kinetics in CX. pipiens oral infection. CX. pipiens mosquitoes were not susceptible to CxFV infection following oral exposure. Columns show infection percentages and the line represents the Ct-values obtained by RT-qPCR. Abbreviation: dpe, days post-exposure

analyzed. The obtained Ct-values were high, indicating low viral load. However, the multiple comparison KruskallWallis test detected significant differences in viral loads among dpi $(H=16.692, d f=5, P=0.005)$. The multiple comparisons of mean ranks indicated that the viral load in bodies of females tested at 7and 9 dpi was significantly higher than at 0 dpi $(z=3.33, P=$ 0.012 and $z=3.06, P=0.033$, respectively), showing CxFV replication within $C x$. pipiens after intrathoracic inoculation (Fig. 2). All saliva samples tested at different time points were negative to $\mathrm{CxFV}$.

CxFV replication kinetics in Cx. pipiens co-infected with RVFV CXFV replication was not affected by RVFV exposure in female Cx pipiens mosquitoes. Results showed that 21 days after CxFV inoculation and 14 days after RVFV exposure (14 dpe), bodies of all tested females remained positive to $\mathrm{CxFV}$ without significant differences (Fig. 3).
RVFV infection, dissemination and transmission in $C x$. pipiens infected and non-infected with CxFV

Mosquitoes infected with $\mathrm{CxFV}$ and exposed to RVFV $(n=10 ; n=1$ hybrid form and $n=9$ molestus form) and mosquitoes non-infected with CxFV and exposed to RVFV $(n=22 ; n=5$ hybrid form and $n=17$ molestus form) were analyzed at $14 \mathrm{dpe}$. The percentages of RVFV infection, dissemination and transmission in analyzed mosquito females were not significantly different between females infected and non-infected with CxFV (Table 1). Moreover, RVFV loads in bodies and legs/wings were not significantly different between females infected and non-infected with CxFV (Fig. 4).

All RVFV-positive saliva were detected in females with disseminated infection at $14 \mathrm{dpe}$. The Ct-values in mosquito saliva did not differ significantly between both groups, infected and non-infected with CxFV (Table 2). In addition, RVFV was detected in bodies, legs/wings or

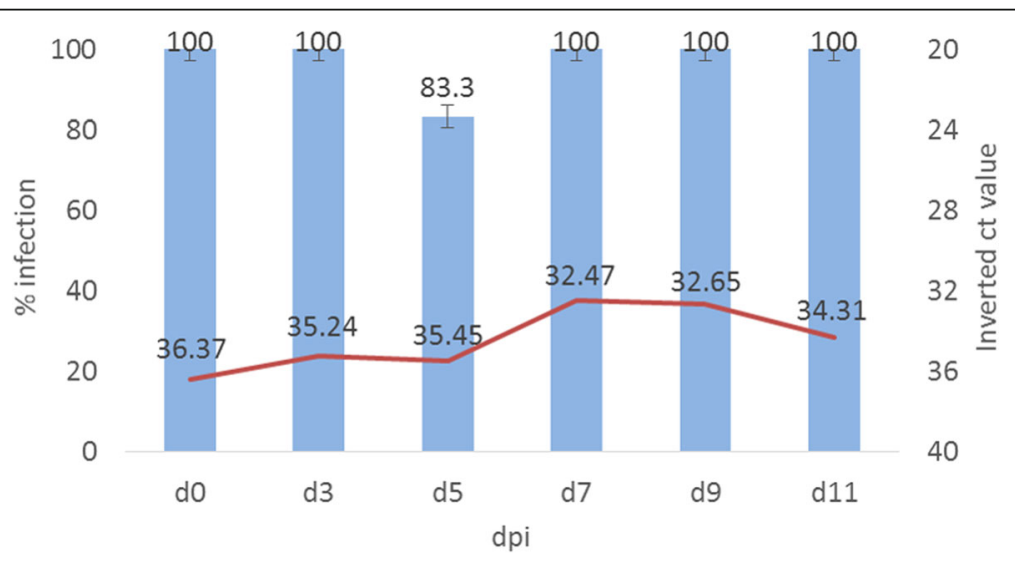

Fig. 2 CXFV replication kinetics in CX. pipiens intrathoracilally inoculated. CX. pipiens mosquitoes were susceptible to CxFV infection after intrathoracic inoculation. Columns show infection percentages and the line represents the Ct-values obtained by RT-qPCR. Abbreviation: dpi, days post-inoculation 


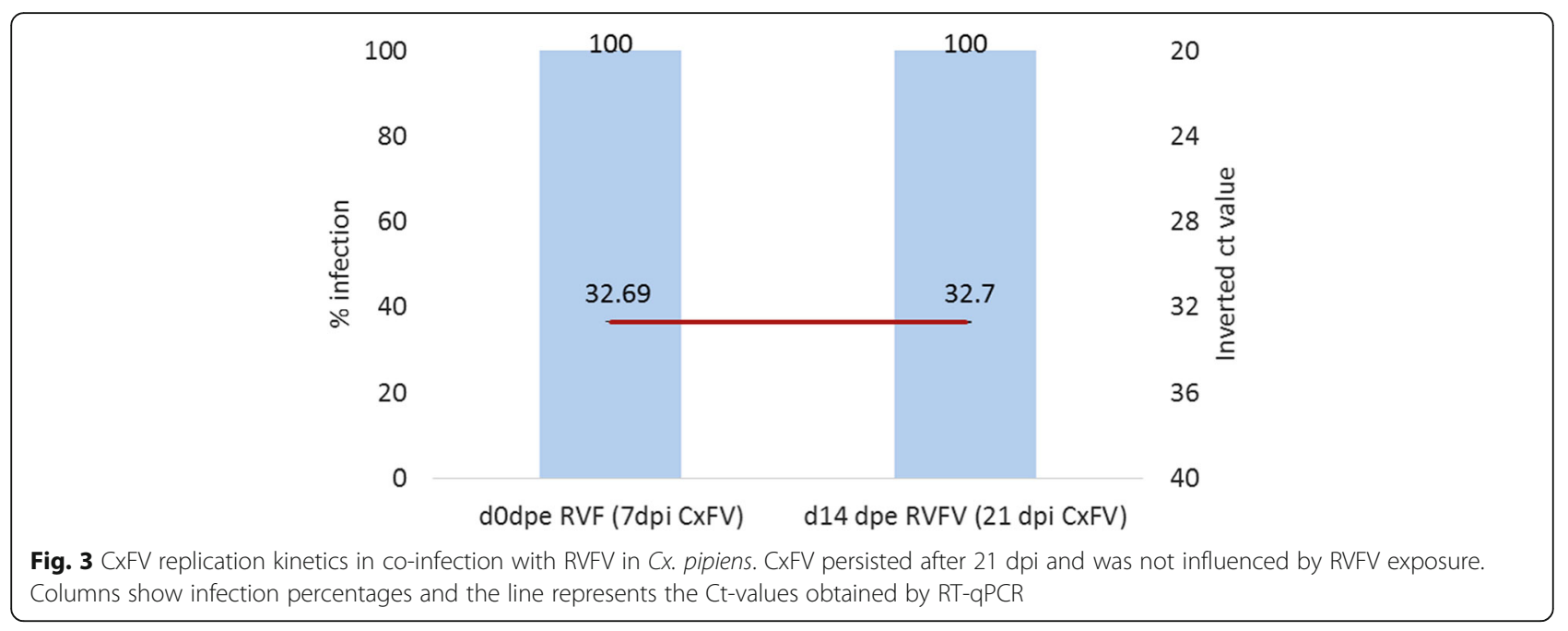

saliva of mosquitoes with $(n=27)$ and without $(n=5)$ Wolbachia (Table 2).

Regarding the forms of individuals from the $C x$. pipiens hybrid colony, RVFV was detected in mosquito bodies, legs/wings and saliva of $C x$. pipiens form molestus and in mosquito bodies of the hybrid form (Table 3).

\section{Discussion}

The isolation, identification and characterization of numerous insect-specific viruses in recent years are of particular interest. They can coexist with pathogenic arboviruses in mosquito populations and may potentially affect the transmission of vector-borne infectious diseases. While there is extensive genetic and phenotypic characterization of insect-specific flaviviruses, little is known about the interactions between them and their mosquito hosts and other arboviruses and the potential public health significance of these associations [39]. Relatively few studies have been performed on co-infections with other flaviviruses such as WNV [18-20]. To the best of our knowledge, the present study is the first to perform a co-infection with two viruses from different genera, CxFV (Flavivirus) and RVFV (Phlebovirus).

The mechanism through which natural mosquito populations become infected with $\mathrm{CxFV}$ is not yet well defined. Our results strongly suggest that $C x$. pipiens females are not susceptible to CxFV upon oral exposure. This is in agreement with previous studies showing transmission of

Table 1 RVFV infection, dissemination and transmission in $C X$. pipiens infected and non-infected with CXFV

\begin{tabular}{lllll}
\hline CxFV infection & IRg & DR & TR & TE \\
\hline+ & $5 / 10(50 \%)$ & $2 / 5(40 \%)$ & $1 / 2(50 \%)$ & $1 / 10(10 \%)$ \\
- & $15 / 22(68 \%)$ & $5 / 15(33 \%)$ & $4 / 5(80 \%)$ & $4 / 22(18 \%)$
\end{tabular}

Abbreviations: $I R$ infection rate, $D R$ disseminated infection rate, $T R$ transmission rate, $T E$ transmission efficiency insect-specific viruses solely among their invertebrate hosts by vertical route $[1,5]$. Intrathoracic inoculation of $\mathrm{CxFV}$ in our study, however, indicates that the virus may have the potential to replicate in Cx. pipiens females at least for 21 days, establishing a possible CxFV persistent infection. Nevertheless, CxFV could not be detected in saliva after $14 \mathrm{dpi}$. Our results are in line with a previous report by Kent et al. [20] who showed that CxFV Izabal intrathoracically inoculated to $C x$. quinquefasciatus females was not found in the saliva.

Vector competence for RVFV was examined at 14 dpe in one $C x$. pipiens colony artificially infected with CxFV by intrathoracic inoculation. The percentage of mosquito females that became infected, developed a disseminated infection, and transmitted RVFV was not significantly different between females infected and non-infected with $\mathrm{CxFV}$. We assume that CxFV may have co-evolved with their mosquito host evading their immune system without affecting its function against a subsequentlyinfecting virus. As such, the molecular mechanisms that allow co-existence of both CxFV and RVFV are not well defined and need more extensive studies. Furthermore, RVFV RNA levels observed were also not significantly different suggesting that CxFV does not affect RVFV replication. This is in agreement with other published studies where co-infection of $\mathrm{CxFv}$ and WNV has been performed. Similarly, Kent et al. [20] investigated the vector competence for WNV of $C x$. quinquefasciatus mosquitoes intrathoracically inoculated with $\mathrm{CxFV}$ Izabal, and also observed no significant differences in WNV titers between CxFV-positive and CxFV-negative mosquitoes at $14 \mathrm{dpi}$. Another study that tested the vector competence for WNV in two Cx. pipiens colonies [19], one colony CxFV naturally infected and the other CxFV non-infected, reported no significant differences in WNV dissemination between both colonies at 14 dpe. However, significant differences were observed at $7 \mathrm{dpe}$, 


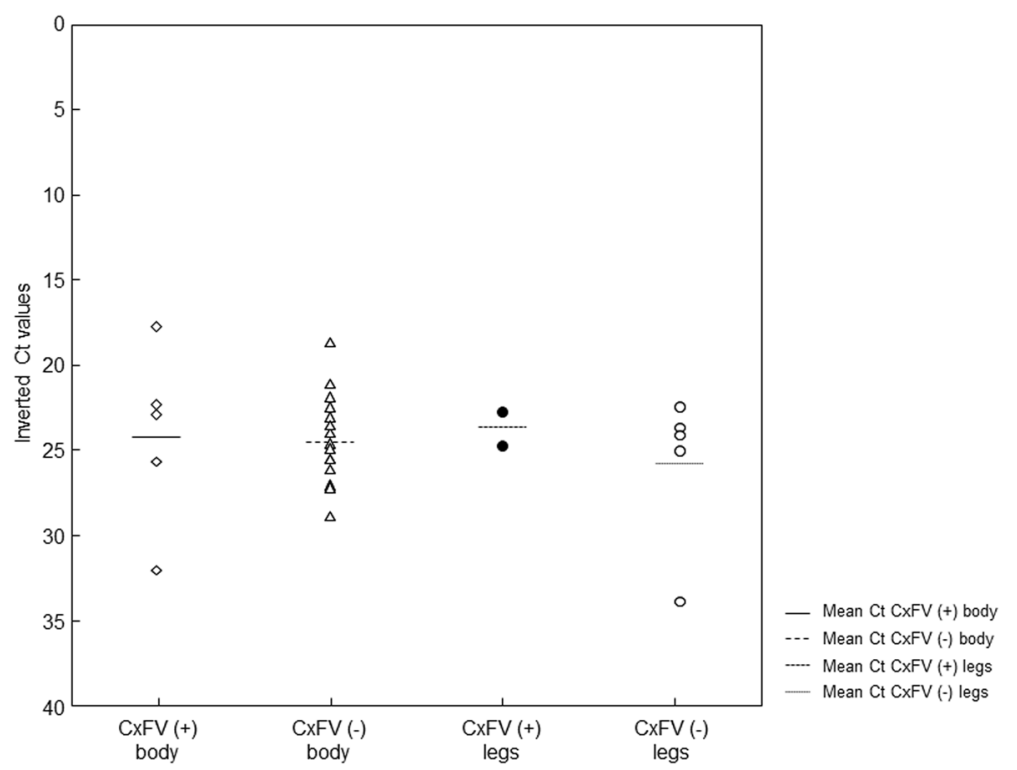

Fig. 4 RVFV Ct-values in female mosquito bodies and legs infected and non-infected with CXFV. RVFV loads in female mosquito bodies and legs/ wings were not affected by CxFV infection

being significantly higher in the CxFV-negative colony than in CxFV-positive colony. These results suggested a competitive interaction between $\mathrm{CxFV}$ and WNV indicating a possible early suppression of WNV replication by $\mathrm{CxFV}$ infection in $C x$. pipiens. Vector competence is influenced by the time-point examined and by genetic differences between mosquito populations [40] as well as genetic diversity and fitness of a laboratory colonized population [41, 42]. All these factors must be taken into account for co-infection studies in mosquitoes.

The $C x$. pipiens colony used in the present study was naturally infected by Wolbachia spp. This may have influenced the vector competence of infected mosquitoes as shown in a previous study [43]. Our results showed that RVFV was detected in bodies, legs/ wings or saliva of mosquitoes with $(n=27)$ and without $(n=5)$ Wolbachia. Due to the small sample size, further studies regarding this issue are needed to explain the potential interference of Wolbachia in arbovirus-vector interactions.

The present study and our previous report [29] allow us to assure that the $C x$. pipiens hybrid colony of Gavà can become infected, disseminate and transmit RVFV. The IR and DIR obtained were lower than those reported by Turrell et al. [44] when a $C x$. pipiens hybrid colony was exposed to a similar RVFV viral dose $\left(10^{7.5} \mathrm{PFU} / \mathrm{ml}\right)$ at $14 \mathrm{dpe}$. Regarding the forms of Culex pipiens, RVFV was detected in mosquito bodies, legs/wings and saliva of $C x$. pipiens form molestus ( $n=26$ tested). Thus, our findings in the present work also showed that the individuals of molestus form within the hybrid colony disseminated and transmitted RVFV. However the virus was only detected in mosquito bodies in hybrid form $(n=6)$. These results may suggest that the individual form might determine the RVFV dissemination and later

Table 2 Presence of RVFV in different samples of mosquitoes with positive saliva at $14 \mathrm{dpe}$. Ct-values of positive samples analysed by RT-qPCR are reported

\begin{tabular}{|c|c|c|c|c|c|}
\hline Individuals & Legs and wings & Saliva & Saliva (CPE) & CxFV & Wolbachia \\
\hline Cx. pipiens molestus & 22.76 & 32.40 & - & 29.49 & - \\
\hline Cx. pipiens molestus & 22.43 & 30.55 & - & - & + \\
\hline Cx. pipiens molestus & 23.70 & 34.13 & - & - & + \\
\hline Cx. pipiens molestus & 24.10 & 32.54 & - & - & + \\
\hline Cx. pipiens molestus & 25.00 & 38.39 & - & - & + \\
\hline
\end{tabular}

Abbreviations: -, negative; +, positive; CPE cytopathic effect 
Table 3 RVFV infection, dissemination and transmission in CX. pipiens molestus form individuals, hybrid form individuals and all individuals of total mosquitoes tested

\begin{tabular}{lllll}
\hline Cx. pipiens & IR & DR & TR & TE \\
\hline Molestus form (individuals) & $16 / 26(61 \%)$ & $7 / 16(44 \%)$ & $5 / 7(71 \%)$ & $5 / 26(19 \%)$ \\
Hybrid form (individuals) & $4 / 6(67 \%)$ & $0 / 4(0 \%)$ & - & $0 / 6(0 \%)$ \\
Total (colony) & $20 / 32(62 \%)$ & $7 / 20(35 \%)$ & $5 / 7(71 \%)$ & $5 / 32(16 \%)$ \\
\hline
\end{tabular}

Abbreviations: $I R$ infection rate, $D R$ disseminated infection rate, $T R$ transmission rate, $T E$ transmission efficiency

transmission, suggesting a strong midgut barrier in hybrid form in $C x$. pipiens individuals.

The insect's immune responses largely determine the viral load, extrinsic incubation period, and mortality of the insect vector after viral infection, all of which directly affect the outcome of disease transmission $[45,46]$. Exposure to one microorganism can provide crossprotection against another microorganism. Specific examples of the super-infection exclusion hypothesis based on the idea of homologous interference, which is the ability of an established infection with one virus to interfere with secondary viral infection, has been documented in cell culture not only with flaviviruses [47-50], but also with other arboviruses of the genera Alphavirus [51], Orbivirus [52] and Vesiculovirus [53, 54]. The study of Bolling et al. [19] reported that CxFV could alter the WNV infection on mosquitoes although it did not exclude WNV infection. However, a positive correlation between WNV and CxFV infection of field-collected $C x$. pipiens mosquitoes from Illinois has been observed, suggesting that there could be a biological suppression that mediates an increasing susceptibility to naturally WNV infected mosquitoes [56]. Moreover, WNV transmission was enhanced in the Honduras colony when mosquitoes were inoculated simultaneously with WNV and CxFV Izabal [20]. To our knowledge, nothing was known about the potential interference of $\mathrm{CxFV}$ in the mosquito infection by other arboviruses not belonging to Flavivirus genus. Our results, for the first time, indicate that $\mathrm{CxFV}$ infection in $C x$. pipiens might not alter the immune system to interfere with the RVFV infection in case of RVFV introduction in $C x$. pipiens populations.

\section{Conclusions}

This is the first study to assess the potential interference of an ISF on RVFV transmission. We have shown that CXFV does not affect RVFV infection, dissemination and transmission. Mosquitoes persistently infected at the assessed conditions may not be used as a preventive intervention strategy for blocking the transmission of RVFV. Further studies using mosquitoes naturally infected with CxFV should be performed to deepen the knowledge in the natural CxFV infection and to elucidate consistent trends for RVFV vector competence in
CxFV artificially and naturally infected $C x$. pipiens populations. Altogether, it is necessary to highlight the importance of deepening the knowledge on the interaction of ISF circulating in mosquito populations present in an area where the potential pathogenic arboviruses can be introduced in order to better assess arbovirus risk transmission. Examining associations between insect-specific viruses such as CxFV and RVFV and other arboviruses important for human and animal health will provide significant new insights into both arbovirus biology and public health.

\section{Abbreviations \\ CXFV: Culex flavivirus; DIR: Disseminated infection rate; DMEM: Dulbecco's modified Eagle medium; FEF: Fully-engorged females; IR: Infection rate; ISFV: Insect-specific flaviviruses; MBFV: Mosquito-borne flaviviruses; RVFV: Rift Valley fever phlebovirus; TE: Transmission efficiency; TR: Transmission rate; WNV: West Nile virus}

\section{Acknowledgments}

The authors would like to thank Dr Carles Aranda, Consell Comarcal del Baix Llobregat, Barcelona, Spain, for providing the mosquito populations and Dr Alejandro Brun for providing the RVFV strain. The authors are very grateful for the excellent technical contributions of Marta Verdún, Núria Pujols and Raquel Rivas from CReSA (IRTA).

\section{Funding}

This project was funded by the CERCA Programme / Generalitat de Catalunya and the Spanish Government (grant no. MINECO AGL2013-47257-P).

Availability of data and materials

All data generated or analysed during this study are included in this published article.

\section{Authors' contributions}

NB conceived and designed the study. AV isolated CxFv from fieldcollected Culex pipiens and tested mosquitoes for Bunyaviridae, Mesoniviridae, Reoviridae, Rhabdoviridae, Togaviridae and Negeviruses viruses. ST, LB and AN performed the experimental assays. FM, ST and NB analyzed the results. NB and ST drafted the manuscript. All authors read and approved the final manuscript.

Ethics approval and consent to participate

Not applicable.

Competing interests

The authors declare that they have no competing interests.

\section{Publisher's Note}

Springer Nature remains neutral with regard to jurisdictional claims in published maps and institutional affiliations. 


\section{Author details}

'IRTA, Centre de Recerca en Sanitat Animal (CReSA, IRTA-UAB), Campus de la Universitat Autònoma de Barcelona, 08193 Bellaterra, Spain. ${ }^{2}$ Departament de Biologia Animal, de Biologia Vegetal i d'Ecologia, Universitat Autònoma de Barcelona, 08193 Bellaterra, Spain. ${ }^{3}$ National Centre for Microbiology, Instituto de Salud Carlos III, Ctra. Majadahonda-Pozuelo, km. 2, 28220 Majadahonda, Madrid, Spain. ${ }^{4}$ CIBER de Epidemiología y Salud Pública. CIBERESP, Instituto de Salud Carlos III, Ctra. Majadahonda-Pozuelo, km. 2, 28220 Majadahonda, Madrid, Spain.

\section{Received: 11 December 2017 Accepted: 6 May 2018}

\section{Published online: 23 May 2018}

\section{References}

1. Hoshino K, Isawa H, Tsuda Y, Yano K, Sasaki T, Yuda M, et al. Genetic characterization of a new insect flavivirus isolated from Culex pipiens mosquito in Japan. Virology. 2007;359:405-14.

2. Moureau G, Ninove L, Izri A, Cook S, de Lamballerie X, Charrel RN. Flavivirus RNA in phlebotomine sandflies. Vector Borne Zoonotic Dis. 2010;10:195-7.

3. Sánchez-Seco MP, Vázquez A, Collao X, Hernández L, Aranda C, Ruiz S, et al. Surveillance of arboviruses in Spanish wetlands: detection of new flavi- and phleboviruses. Vector Borne Zoonotic Dis. 2010;10:203-6.

4. Lutomiah JJ, Mwandawiro C, Magambo J, Sang RC. Infection and vertical transmission of Kamiti river virus in laboratory bred Aedes aegypti mosquitoes. J Insect Sci. 2007;7:1-7.

5. Sang RC, Gichogo A, Gachoya J, Dunster MD, Ofula V, Hunt AR, et al. Isolation of a new flavivirus related to cell fusing agent virus (CFAV) from field-collected flood-water Aedes mosquitoes sampled from a dambo in central Kenya. Arch Virol. 2003;148:1085-93.

6. Calzolari $M$, Zé-Zé L, Růžek D, Vázquez $A$, Jeffries $C$, Defilippo F, et al. Detection of mosquito-only flaviviruses in Europe. J Gen Virol. 2012;93:1215-25.

7. Cerutti F, Giacobini M, Mosca A, Grasso I, Rambozzi L, Rossi L, et al. Evidence of mosquito-transmitted flavivirus circulation in Piedmont, north-western Italy. Parasit Vectors. 2012:5:99.

8. Osório HC, Zé-Zé L, Amaro F, Alves MJ. Mosquito surveillance for prevention and control of emerging mosquito-borne diseases in Portugal - 2008-2014. Int J Environ Res Public Health. 2014;11:11583-96.

9. Papa A, Papadopoulou E, Kalaitzopoulou S, Tsioka K, Mourelatos S. Detection of West Nile virus and insect-specific flavivirus RNA in Culex mosquitoes, central Macedonia, Greece. Trans R Soc Trop Med Hyg. 2014; 108(9):555.

10. Vazquez A, Sanchez-Seco MP, Palacios G, Molero F, Reyes N, Ruiz S, et al Novel flaviviruses detected in different species of mosquitoes in Spain. Vector Borne Zoonotic Dis. 2012;12:223-9.

11. Datta S, Gopalakrishnan R, Chatterjee S, Veer V. Phylogenetic characterization of a novel insect-specific flavivirus detected in a Culex pool, collected from Assam, India. Intervirology. 2015;58:149-54.

12. Hoshino K, Takahashi-Nakaguchi A, Isawa H, Sasaki T, Higa Y, Kasai S, et al. Entomological surveillance for flaviviruses at migratory bird stopover sites in Hokkaido, Japan, and a new insect flavivirus detected in Aedes galloisi (Diptera: Culicidae). J Med Entomol. 2012;49:175-82.

13. Ochieng C, Lutomiah J, Makio A, Koka H, Chepkorir E, Yalwala S, et al. Mosquito-borne arbovirus surveillance at selected sites in diverse ecological zones of Kenya, 2007-2012. Virol J. 2013;10:140.

14. Pabbaraju K, Ho KCY, Wong S, Fox JD, Kaplen B, Tyler S, et al. Surveillance of mosquito-borne viruses in Alberta using reverse transcription polymerase chain reaction with generic primers. J Med Entomol. 2009;46:640-8.

15. Alba A, Allepuz A, Napp S, Soler M, Selga I, Aranda C, et al. Ecological surveillance for West Nile in Catalonia (Spain), learning from a five-year period of follow-up. Zoonoses Public Health. 2014;61:181-91.

16. Aranda C, Sanchez-Seco MP, Caceres F, Escosa R, Galvez JC, Masia M, et al. Detection and monitoring of mosquito flaviviruses in Spain between 2001 and 2005. Vector Borne Zoonotic Dis. 2009:9:171-8.

17. Crabtree MB, Sang RC, Stollar V, Dunster LM, Miller BR. Genetic and phenotypic characterization of the newly described insect flavivirus, Kamiti River virus. Arch Virol. 2003:148:1095-118.

18. Goenaga S, Kenney JL, Dugal NK, Delorey M, Elbel GD, Zhang B, et al. Potential for co-infection of a mosquito-specific flavivirus, Nhumirim virus, to block West Nile virus transmission in mosquitoes. Viruses. 2015;7:5801-12.

19. Bolling BG, Olea-Popelka FJ, Eisen L, Moore CG, Blair CD. Transmission dynamics of an insect-specific flavivirus in a naturally infected Culex pipiens laboratory colony and effects of co-infection on vector competence for West Nile virus. Virology. 2012;427:90-7.

20. Kent RJ, Crabtree MB, Miller BR. Transmission of West Nile virus by Culex quinquefasciatus Say infected with Culex flavivirus Izabal. PLoS Negl Trop Dis. 2010;4:e671

21. Olive MM, Goodman SM, Reynes JM. The role of wild mammals in the maintenance of Rift Valley fever virus. J Wildl Dis. 2012;48:241-66.

22. Daubney R, Hudson JR, Garnham PC. Enzootic hepatitis or rift valley fever An undescribed virus disease of sheep cattle and man from East Africa. J Pathol Bacteriol. 1931;34:545-79.

23. Nanying MO, Munyua P, Kiama SG, Muchemi GM, Thumbi SM, Bitek AO, et al. A systematic review of Rift Valley fever epidemiology 1931-2014. Infect Ecol Epidemiol. 2015:5:28024.

24. Ahmad K. More deaths from Rift Valley fever in Saudi Arabia and Yemen. Lancet. 2000:356:1422.

25. Chevalier V, Pepin M, Plee L, Lancelot R. Rift Valley fever - a threat for Europe? Euro Surveill. 2010;15:19506.

26. Mansfield KL, Banyard AC, McElhinney L, Johnson N, Horton DL, HernándezTriana LM, et al. Rift Valley fever virus: a review of diagnosis and vaccination, and implications for emergence in Europe. Vaccine. 2015;33:5520-31.

27. Rolin Al, Berrangeford L, Kullkarni MA. The risk of Rift Valley fever virus introduction and establishment in the United States and European Union. Emerg Microbes Infect. 2013;2:e81.

28. Sánchez- Vizcaino F, Martinez-Lopez B, Sánchez-Vizcaino JM. Identification suitable areas for the occurrence of Rift Valley fever outbreaks in Spain using a multiple criteria decision framework. Vet Microbiol. 2013;165:71-8.

29. Brustolin M, Talavera S, Nuñez A, Santamaría C, Rivas R, Pujol N, et al. Rift Valley fever virus and European mosquitoes: vector competence of Culex pipiens and Stegomyia albopicta (= Aedes albopictus). Med Vet Entoml. 2017; 31:365-72.

30. Abdo-Salem S, Waret-Szkuta A, Roger F, Olive MM, Saeed K, Chevalier V. Risk assessment of the introduction of Rift Valley fever from the Horn of Africa to Yemenvia legal trade of small ruminants. Trop Anim Health Prod. 2011; 43:471-80.

31. Turell MJ, Presley SM, Gad AM, Cope SE, Dohm DJ, Morrill JC, et al. Vector competence of Egyptian mosquitoes for Rift Valley fever virus. Am J Trop Med Hyg. 1996;54:136-9.

32. Amraoui F, Krida G, Bouattour A, Rhim A, Daaboub J, Harrat Z, et al. Culex pipiens, an experimental efficient vector of West Nile and Rift Valley fever viruses in the Magreb region. PLoS One. 2012;7:e36757.

33. Bahnck CM, Fonseca DM. Rapid assay to identify the two genetic forms of Culex (Culex) pipiens L. (Diptera: Cilicidae) and hybrid populations. Am J Trop Med Hyg. 2006;75:251-5.

34. Vasilakis N, Tesh RB. Insect-specific viruses and their potential impact on arbovirus transmission. Curr Opin Virology. 2015:15:69-74.

35. Zhou W, Rousset F, O'Neill S. Phylogeny and PCR-based classification of Wolbachia strains using wsp gene sequences. Proc R Soc. 1998;265:509-15.

36. Villegas P. Titration of biological suspensions. In: Swayne DE, Glisson JR, Jackwood MW, Pearson JE, Reed WM, editors. A laboratory manual for the isolation and identification of avian pathogens. 4th ed. Kennet Square, Pennsylvania: The American Association of Avian Pathologists Inc; 1998. p. 248-54.

37. Busquets N, Xavier F, Martín-Folgar R, Lorenzo G, Galindo-Cardiel I, del Val $\mathrm{BP}$, et al. Experimental infection of young adult European breed sheep with Rift valley fever virus field isolates. Vector Borne Zoonotic Dis. 2010;10:698-6.

38. Chouin-Carneiro T, Vega-Rua A, Vazeille M, Yebakima A, Girod R, Goindin D, et al. Differential susceptibilities of Aedes aegypti and Aedes albopictus from the Americas to Zika Virus. PLoS Negl Trop Dis. 2016;10:e0004543.

39. Crockett RK, Burkhalter K, Mead D, Kelly R, Brown J, Varnado W, et al. Culex flavivirus and West Nile virus in Culex quinquefasciatus populations in the southeastern United States. J Med Entomol. 2012:49:165-74.

40. Bennett KE, Olson KE, Muñoz M, Fernandez-Salas I, Farfan-Ale JA, Higgs S, et al. Variation in vector competence for dengue 2 virus among 24 collections of Aedes aegypti from Mexico and the United States. Am J Trop Med Hyg. 2002;67:85-92.

41. Lambrechts L, Scott TW, Gubler DJ. Consequences of the expanding global distribution of Aedes albopictus for dengue virus transmission. PLoS Negl Trop Dis. 2010:4:e646.

42. Lorenz L, Beaty BJ, Aitken THG, Wallis GP, Tabachnick WJ. The effect of colonization upon Aedes aegypti susceptibility to oral infection with yellow fever virus. Am J Trop Med Hyg. 1984;33:690-4. 
43. Moreira LA, Iturbe_Ormaetxe I, Jeffery JA, Lu G, Pyke AT, Hedges LM, et al. A Wolbachia symbiont in Aedes aegypti limits infection with dengue, chikungunya, and Plasmodium. Cell. 2009;139:1268-78.

44. Turell MJ, Dohm DJ, Fonseca DM. Comparison of the potential for different genetic forms in the Culex pipiens complex in north America to transmit Rift Valley fever virus. J Am Mosq Control Assoc. 2014;320:253-9.

45. Ocampo CB, Caicedo PA, Jaramillo G, Ursic Bedoya R, Baron O, Serrato IM, et al. Differential expression of apoptosis related genes in selected strains of Aedes aegypti with different susceptibilities to dengue virus. PLoS One. 2013;8:e61187.

46. Sim S, Jupatanakul N, Dimopoulos G. Mosquito immunity against arboviruses. Viruses. 2014;6:4479-504.

47. Burivong P, Pattanakitsakul SN, Thongrungkiat S, Malasit P, Flegel TW. Markedly reduced severity of dengue virus infection in mosquito cell cultures persistently infected with Aedes albopictus densovirus (AalDNV). Virol. 2004;329:261-9.

48. Pepin KM, Lambeth K, Hanley KA. Asymmetric competitive suppression between strains of dengue virus. BMC Microbiol. 2008;8:28-37.

49. Randolph VB, Hardy JL. Establishment and characterization of St Louis encephalitis virus persistent infections in Aedes and Culex mosquito cell lines. J Gen Virol. 1988;69:2189-98.

50. Sundin DR, Beaty BJ. Interference to oral superinfection of Aedes triseriatus infected with La Crosse virus. Am J Trop Med Hyg. 1988;38:428-32.

51. Karpf AR, Lenches E, Strauss EG, Strauss JH, Brown DT. Superinfection exclusion of alphaviruses in three mosquito cell lines persistently infected with Sindbis virus. J Virol. 1997:71:7119-23.

52. Ramig RF, Garrison C, Chen D, Bell-Robinson D. Analysis of reassortment and superinfection during mixed infection of Vero cells with bluetongue virus serotypes 10 and 17. J Gen Virol. 1989;70:2595-603.

53. Legault D, Takayesu D, Prevec L. Heterotypic exclusion between vesicular stomatitis viruses of the New Jersey and Indiana serotypes. J Gen Virol. 1977; 35:53-65.

54. Whitaker-Dowling P, Youngner JS, Widnell CC, Wilcox DK. Superinfection exclusion by vesicular stomatitis virus. Virology. 1983;131:137-43.

\section{Ready to submit your research? Choose BMC and benefit from:}

- fast, convenient online submission

- thorough peer review by experienced researchers in your field

- rapid publication on acceptance

- support for research data, including large and complex data types

- gold Open Access which fosters wider collaboration and increased citations

- maximum visibility for your research: over $100 \mathrm{M}$ website views per year 